\section{Agricultura y desarrollo local en Guatemala}

RESUMEN

Este artículo estudia la función de la agricultura como motor de desarrollo local. Para el efecto, se analiza la experiencia de cuatro municipios de Guatemala, dos donde se practica agricultura de gran extensión y dos de esta a pequeña escala. Los resultados muestran diferencias entre ambos grupos. En el primero, el nivel de pobreza se mantuvo alto, en tanto que en el segundo, la tasa de esta se redujo en un monto significativo durante un período de seis años. Los municipios difieren en ubicación geográfica, tamaño y composición étnica, pero tienen en común la agricultura como principal actividad productiva.

Palabras Clave

Agricultura; desarrollo; desarrollo local; pobreza; capital.

\section{Local Development Agriculture in Guatemala}

ABSTRACT

This article examines the role of agriculture as an engine of local development. To this end, the experience of four municipalities in Guatemala, where two large agricultural extension and two practiced small-scale agriculture is analyzed. The results show differences between groups. In the first the poverty rate remained high, while in the second the poverty rate fell by a significant amount over a period of six years. Municipalities differ in geographic location, size and ethnic composition, but have in common agriculture as main activity.

\section{KEY WORDS}

Agriculture; development; local development; poverty; capital.
Artículo recibido:

22 de agosto del 2014.

Evaluado:

7 de octubre del 2014.

Aceptado:

3 de noviembre del 2014.

Economista guatemalteco, Doctor en sociología. Profesor de la Universidad Rafael Landívar, e investigador del Instituto de Investigaciones Económicas (IDIES) de esta misma universidad en Guatemala. Tiene maestría en Docencia Universitaria por la Universidad Rafael Landívar y Licenciatura en Economía por la Universidad de San Carlos de Guatemala. Correo electrónico: godiaz@url.edu.gt 


\section{Agricultura y desarrollo local en Guatemala}

La agricultura en Guatemala representa el 12\% del Producto Interno Bruto y participa con casi $33 \%$ del empleo y $25 \%$ de las exportaciones (CEPAL, 2013). Las mujeres participan en el 19\% del empleo agrícola (Instituto Nacional de Estadística [INE], 2010). La participación de ellas como productoras es del 8\%, según datos del IV Censo Agropecuario (Instituto Nacional de Estadística, 2004), menor a la participación como trabajadora. De igual manera, también es limitado el acceso a la tierra por parte de las féminas, un estudio realizado en la cuenca Ostúa-Güija en el oriente del país revela que solo $13 \%$ de los entrevistados con acceso a la tierra son mujeres (Pelaez, 2011).

En el país, alrededor de la mitad de la población vive en condiciones de pobreza. Sin embargo, a nivel geográfico se observan polos de desarrollo, donde la pobreza es menor. Este artículo explora la relación entre agricultura y desarrollo, en especial el desarrollo local, a partir de comparar la situación de cuatro municipios, en los cuales la agricultura es una de sus principales actividades económicas.

\section{Revisión literaria}

En la teoría del crecimiento económico, son varios los autores, entre ellos Rostow (1961) y Kaldor (Thirwall, 2003), que restan importancia a la agricultura y resaltan la industria como motor de desarrollo. La teoría elaborada por Rostow asigna a la agricultura la función de proveer alimentos y demandar productos industriales; acá la fase de despegue del crecimiento se alcanza a través de la industria. La teoría de Kaldor argumenta que existe fuerte relación de causalidad entre el crecimiento industrial y el económico. 
Por su parte, el modelo de crecimiento de Harrod-Domar se basa en los aportes de los factores productivos trabajo y capital. Este postula que solo a través de un aumento de la tasa de inversión se obtiene mayor crecimiento económico y que los rendimientos decrecientes de la agricultura lo obstaculizan (Zimmerman, 1970). Por último, el modelo de crecimiento de Solow (1956) también considera los factores trabajo y capital, pero les agrega la tecnología. De nuevo, se excluye el factor tierra ligado a la agricultura, para explicar el crecimiento económico. Estos y otros modelos comparten la premisa de la acumulación de capital físico, representado de forma preferente en la industria, como lo que genera crecimiento económico.

Durante la segunda mitad de la pasada década, cambió la visión respecto a la relación entre agricultura y crecimiento económico. Estudios realizados en las décadas de 1960 y 1970 evidenciaron que el crecimiento de la productividad agrícola contribuye al económico por medio de oferta de alimentos, generación de divisas y empleo, y transferencia de capital hacia el sector industrial (Bejarano, 1998).

Entre los diversos autores que favorecen la premisa de la agricultura como fuente de desarrollo económico, se puede mencionar a Schultz (1967), quien abogó por la transformación de la agricultura tradicional a través de la aplicación de la ciencia y tecnología en los métodos de producción agrícola. Según Schultz, el agricultor que siembra igual a sus antepasados no produce más que ellos, aunque la tierra sea muy fértil. El uso de la tecnología en la agricultura, sostiene, permite aumentar la productividad y los ingresos de los productores. Douglas North (Prado, 1998) es otro autor a favor de la agricultura como motor de crecimiento económico. A su criterio, la agricultura de exportación puede propiciarlo.

El nexo entre agricultura y crecimiento ha sido respaldado empíricamente en diversos países (Banco Mundial, 2008). Un estudio realizado en 62 países para el período 1960 a 1990 evidenció que el aumento en la productividad agrícola es importante para explicar el crecimiento del PIB por habitante (Diao, Hazell, Resnick y Thurlow, 2006). Más en concreto, un incremento de $1 \%$ en el ingreso del PIB agrícola por habitante genera un aumento de 1,61\% en el ingreso del quintil más pobre de la población (Timmer, 1997. Citado en Mellor, 2000). La experiencia también revela la contribución de la agricultura al combate de la pobreza (Thomson, 2004; Dethier y Effenberger, 2011). Un estudio de la FAO (1995) presenta datos sobre la reducción de la pobreza rural en diversos países. El mecanismo a través del cual se da lo anterior es el aumento del empleo (Dixon y Gulliver, 2001).

Al igual que cambió la visión del nexo entre agricultura y desarrollo, también cambió la concepción sobre este último. Hacia la década de 1950, se concebía como crecimiento económico; debido la expansión de la población, a través del tiempo se consideró que para aumentar el ingreso por habitante era necesario generar un crecimiento económico mayor que el de la población (Meier y Stiglitz, 2001). Sin embargo, esta visión del desarrollo comenzó a 
ser cuestionada décadas después, por considerar que el desarrollo no podía reducirse solo a aumento del ingreso por habitante. Además, esta variable como indicador de desarrollo era imperfecta, pues el ingreso no se distribuye de forma uniforme entre la población.

En consecuencia, a principios de la década de 1990 se comenzó a hablar de desarrollo humano, más que del económico. El desarrollo humano fue concebido, en sus inicios, como un proceso que permitía incrementar las oportunidades de las personas y vivir mejor (Quan, 2007). En este caso, la medición se da a través del Índice de Desarrollo Humano (IDH), este combina las variables ingreso, educación y esperanza de vida; su ventaja radica en combinar importantes indicadores en materia de desarrollo. El valor del índice varía entre 0 y 1 y señala el grado de desarrollo alcanzado (Ray, 2002).

En lo que respecta al ámbito geográfico, la perspectiva económica sobre el desarrollo cambió en las últimas tres décadas, pues pasó de concebirse como un proceso a nivel macroeconómico, es decir, nacional, a uno territorial. En este sentido, el desarrollo pasó de verse solo "desde arriba", producido por la gran empresa, a considerarse "desde abajo", con base en recursos endógenos y realizado por la pequeña empresa (Alburquerque, 2003). A partir de allí, la teoría incorporó durante ese periodo el concepto de desarrollo económico local, el cual es entendido como un proceso de crecimiento económico y cambio estructural, que mejora el nivel de bienestar de una localidad o región. Cuando la comunidad lidera ese proceso de cambio estructural se está ante un desarrollo local endógeno (Vásquez, 2007).

El Banco Mundial (Swinburn, Goga, y Murphy, 2006) define el desarrollo económico local como un proceso en el que diversos sectores de la sociedad trabajan en conjunto para mejorar condiciones de crecimiento económico y generación de empleo. Una definición simple, pero de amplio significado, es la utilizada por Local Government Comission -Asociación de Gobierno Local en los Estados Unidos de América- para la cual desarrollo significa mejorar en la calidad de vida (Pike, Rodríguez y Tomaney, 2011). Así, los componentes de este son territorio, sociedad y cultura, y los actores están constituidos por la población, las organizaciones, el gobierno local, las empresas y otros actores sociales (Díaz y Ascoli, 2006).

El debate sobre el desarrollo está en la agenda de diversos actores económicos, sociales y políticos de Guatemala. El sector empresarial lo identifica con crecimiento económico y se adhiere a la tradición de la teoría del derrame; según esta, basta con generar crecimiento económico para que se produzca desarrollo. Dicha perspectiva considera la inversión privada como base para el desarrollo del país, pues esta origina crecimiento económico, lo que a su vez se traduce en empleo y mejoras en el nivel de ingreso de la población. La visión empresarial sostiene que con mayor crecimiento, menor será la pobreza (larna, 2013). Sin embargo, esto es cuestionable porque pese al crecimiento económico evidenciado, el país sigue teniendo una alta tasa de pobreza. Otra crítica es que enfatiza el crecimiento sin considerar su sostenibili- 
dad, tal es el caso de las actividades extractivas; por ejemplo, la minería, la cual depende de los recursos mineros. En ese sentido, se crea un auge productivo insostenible.

Por su parte, el sector cooperativista tiene una visión del desarrollo similar al empresarial, pero cuestiona la desigual distribución de riqueza, manifiesta en el alto coeficiente de Gini que tiene el país; esto a su vez incide en el bajo desarrollo humano de la mayoría de la población. Entonces, para revertirlo, se propicia la asociación cooperativa en las diversas fases del ciclo productivo, con el objetivo de generar una acumulación horizontal de la riqueza (ARNA, 2013). Esta visión también es cuestionable por asumir, en parte, que el crecimiento económico es suficiente para generar desarrollo. En este sentido, su planteamiento propone que el país fomente sus actuales actividades productivas, tales como minería, agroexportación, maquila textil, comercio y servicios. Por lo tanto, aunque se adhiere al enfoque de la economía social para mejorar la distribución de la riqueza, el aporte cooperativista es limitado pues abarca alrededor del $10 \%$ de la población total.

La perspectiva del Estado guatemalteco, a través del plan de desarrollo gubernamental Ilamado "K`atun 2032", se enmarca en la visión de crecimiento económico, pero adiciona elementos de desarrollo rural, social y humano. Dicha propuesta incorpora lo mejor de distintos enfoques, incluyendo el desarrollo sostenible. Sin embargo, se mantiene dentro de la tradición de la teoría del derrame y la explotación de los recursos naturales, con lo cual se logra el crecimiento económico a través de los sectores agrícola, minero y forestal, entre otros (IARNA, 2013). Desde el punto de vista metodológico, esta visión de desarrollo es una mezcla de enfoques, aspecto que debilita su fundamentación.

En cuanto a los sectores laboral, campesino, ambiental y civil, estos son partidarios del desarrollo sostenible, con características propias del país, en especial en lo referente a la economía familiar campesina como motor de desarrollo (IARNA, 2013). En esta investigación, el enfoque de desarrollo local sobre la base de la agricultura a pequeña escala se aproxima más a esta visión.

Como ya se dijo en Guatemala predomina el enfoque de crecimiento económico, fundamentado en la teoría del derrame y en una perspectiva macroeconómica. No obstante, el desarrollo desde la perspectiva local es un enfoque poco extendido, en el mejor de los casos se habla de desarrollo territorial o regional, pues abarca amplias extensiones de territorio. En cuanto al enfoque de desarrollo humano, está subordinado al carácter de crecimiento económico.

Los desarrollos humano y económico local tienen en común la idea de mejora en la calidad de vida de la población, esta es la perspectiva adoptada en este artículo para tratar el desarrollo. Se considera que los indicadores socioeconómicos de pobreza e IDH son útiles para medir las mejoras en calidad de vida. 
El IDH es un indicador muy conocido en los estudios sobre el desarrollo y su definición es uniforme. El concepto de pobreza varía según el contexto en que se aplique, por lo cual conviene hacer algunas acotaciones sobre este para el caso de Guatemala. En este país, se refiere (con frecuencia) a la falta de recursos para obtener los bienes y servicios necesarios para tener un nivel de vida aceptable. Desde esta perspectiva, se entiende la pobreza como un concepto material, carencia de recursos y como una situación económica asociada a la falta de ingresos. En la práctica, la pobreza se mide bajo el enfoque de la línea de pobreza, que representa el monto de dinero que se necesita para tener dicho nivel de vida. En la última medición oficial, la línea de pobreza en Guatemala se fijó en Q8.283,00, equivalente a casi \$1.175,00, por persona al año. El enfoque multidimensional en esta nación es poco difundido y se encuentra ausente en las mediciones oficiales de dicho fenómeno económico y social. Los datos municipales utilizados en este trabajo corresponden a la medición oficial de 2006.

\section{Agricultura en Guatemala}

La agricultura en Guatemala puede clasificarse entre la de gran extensión y la de pequeña escala. Por la primera debe entenderse aquella que se práctica en unidades productivas de gran tamaño y, en general, vinculada con productos tradicionales de exportación, tales como azúcar y banano. En términos de organización económica, es una agricultura comercial basada en la gran empresa. Por su parte, la de pequeña escala se refiere, en esta investigación, a aquella realizada en una unidad productiva de reducido tamaño, por lo general, menor a 10 hectáreas, cuya producción se orienta al mercado interno o externo. Esta se basa en la pequeña empresa, con frecuencia de propiedad familiar y abarca cultivos de maíz, frijol y hortalizas.

En cuanto a la agricultura de gran extensión, a causa de la fertilidad del suelo y las condiciones climáticas, el país se ubica entre los principales productores a nivel mundial de café, banano y azúcar. En el caso del primero, según datos de la Organización Internacional de Café (2014), Guatemala es el décimo exportador, con el $2 \%$ del mercado mundial, después de Honduras, con el $3 \%$. En banano, es el quinto exportador mundial, después de Costa Rica y Colombia que ocupan las posiciones tres y cuatro respectivamente, según el volumen exportado (FAO, 2014). En azúcar, ocupa el cuarto puesto de exportador mundial, después de Brasil, Tailandia y Australia (Agrequimia, 2012).

En años recientes, el sector agrícola experimentó importantes inversiones nacionales y extranjeras, en la expansión de los cultivos caña de azúcar, banano, palma africana y hule (ver Tabla 1). La producción de estos se ha dado a gran escala. Por ejemplo, en 2003 el tamaño promedio de la finca de palma africana fue de 633 hectáreas, de hule 38,8 hectáreas y de caña de azúcar 13,3 hectáreas. En lo que respecta a la agricultura a pequeña escala, se refiere a la relacionada con productos de consumo interno, como el maíz y frijol, y de exportación, como hortalizas, en áreas de poca dimensión. El tama- 
ño promedio de una finca de maíz fue de 0,95 hectáreas en 2003, mientras que en la última década, se expandió en especial la producción de este grano y del frijol (INE, 2004).

\begin{tabular}{lccc}
\hline & \multicolumn{3}{c}{ Tabla 1 } \\
& Área cosechada en hectáreas & \\
\hline \multicolumn{1}{c}{ Cultivo } & $\mathbf{2 0 0 6}$ & $\mathbf{2 0 1 1}$ & Variación \\
\hline Café & 247365 & 252416 & 5051 \\
\hline Azúcar & 188702 & 239261 & 50559 \\
\hline Hule & 114200 & 225000 & 110800 \\
\hline Palma africana & 43000 & 100000 & 57000 \\
\hline Banano & 42377 & 64289 & 21912 \\
\hline Maíz & 577249 & 841049 & 263800 \\
\hline Frijol & 220152 & 238140 & 17988 \\
\hline
\end{tabular}

Fuente: MAGA, 2011 y Agrequimia, 2012.

En conjunto, el área cosechada en cultivos de agricultura de gran extensión creció en 38\% durante el quinquenio 2006 a 2011, contra 35\% de tasa de crecimiento registrada por los dos principales cultivos para la alimentación, maíz y frijol, de pequeña escala. Sin embargo, en términos absolutos, estos últimos aumentaron su área cosechada en mayor monto que los primeros. Dicho aumento se explica por la expansión de la frontera agrícola. En el caso del hule, debido a su aumento en la demanda externa, Guatemala se posiciona como el octavo exportador mundial, y en el caso del maíz y frijol ocurre por el incremento de la población (Agrequimia, 2012).

Según lo mencionado, los cultivos de banano, caña de azúcar, hule y palma africana han tenido una amplia expansión geográfica en Guatemala. El mayor porcentaje de la producción de banano se cosecha en los departamentos de Escuintla, con 46\% del total, e Izabal, con 33\%. De acuerdo con información del Ministerio de Economía (2010), el cultivo de esta fruta es realizado por grandes empresas, por consiguiente hay un número diminuto de pequeños productores. Guatemala es el octavo exportador de banano a nivel mundial, así su producción se destina a exportar; Estados Unidos es el principal mercado, con más del $80 \%$ de esta. Su cultivo genera 62.000 empleos en forma directa y 10000 indirectos.

En cuanto a la caña de azúcar, Guatemala cuenta con 469.000 hectáreas disponibles para su cultivo. De ese total, para el año 2011 eran cultivadas alrededor de 240.000 , lo cual equivale al 51\%. El 70\% de la producción de azúcar se destina a las exportaciones, en especial al mercado de Estados Unidos y de países asiáticos. Esta expansión se da en el país debido a su alta 
competitividad a nivel mundial, así es el tercer productor en el mundo. Los dos principales productores son Brasil y Tailandia.

El cultivo del hule está aumentando en Guatemala, el área cosechada se duplicó entre 2006 y 2011, de 114.000 a 225.000 hectáreas. Este fenómeno se debe a las favorables condiciones agroecológicas, al punto que Guatemala es el cuarto productor mundial de esta materia prima (Agrequimia, 2012). La producción exporta el $62 \%$ a México, seguido de Costa Rica y Colombia, cada país con un $12 \%$ de las exportaciones.

La palma africana registró gran expansión en el quinquenio 2006 a 2011, con un aumento de 57.000 hectáreas cultivadas, (MAGA, 2011). Tal expansión ha sido fomentada gracias a los altos rendimientos por hectárea que el país tiene en el cultivo de este producto, los cuales lo ubican como el exportador número once a nivel mundial (Agrequimia, 2012). Adicionalmente, existen otros cultivos que se expandirán a futuro, tal es el caso del piñón o Jatropha, promovido por el MAGA para la producción de biocombustibles, y el de teca, promovido por la Gremial Forestal.

Del total de productores agrícolas (830.684) registrados en el censo agrícola de 2003 (Instituto Nacional de Estadística, 2004), el 92\% equivalente a 764.685, eran pequeños productores, con una extensión de tierra menor a siete hectáreas, la cual, en la mayoría de casos, solo les permite practicar una agricultura de subsistencia.

Los dos principales cultivos a pequeña escala son el maíz y el frijol, estos tienen una importancia vital en la dieta de los guatemaltecos. lo mismo puede decirse de la papa y el tomate. Impulsados debido a la demanda externa, se ha desarrollado la producción de café a pequeña escala, así también de cardamomo, mango, piña, brócoli, ejote francés, chile pimiento, lechuga, zanahoria y otras frutas y legumbres. La producción de maíz se da para consumo interno, sin embargo, su volumen resulta insuficiente, por lo cual se requiere importar. El consumo interno en el año 2010 fue de 49,5 millones de quintales, de los cuales la producción nacional abarcó 35,3 millones. La productividad promedio del maíz es de 2,1 toneladas métricas por hectárea (Agrequimia, 2012).

Por su parte, el cultivo de frijol acaparó casi 240.000 hectáreas en el año 2011, con una producción de 215.990 toneladas métricas, su productividad es de 0,90 toneladas métricas por hectárea. Al igual que el maíz, la producción de este grano es para el consumo interno, pero resulta insuficiente, por ello, se debe importar. En cuanto al café y al cardamomo, del primero un alto porcentaje de pequeños productores está organizado en la Federación de Cooperativas Agrícolas Productoras de Café de Guatemala (Fedecocagua), que agrupa a casi 150 cooperativas, con cerca de 20.000 miembros. Por su parte, el Gremial de Exportadores de Cardamomo concentra la oferta exportable de los más de 100.000 pequeños agricultores de este cultivo. 
En el cultivo de mango, Guatemala es uno de los países con mayor productividad, aporta 26 toneladas métricas por hectárea, aunque no figura entre los productores comerciales registrados en Unctad. En el país existen alrededor de 42.000 productores de mango con un área cosechada de 13.000 hectáreas (CABI, 2011). Su producción es tanto para consumo local como para exportación. También se utiliza como insumo por parte de la industria alimenticia en la elaboración de jugos y mermeladas.

Los cultivos arveja china, ejote francés y brócoli son orientados a la exportación, principalmente a Estados Unidos y países europeos. Guatemala es el segundo exportador mundial de los primeros dos producto y el número ocho en brócoli. La producción de arveja está integrada por agrupaciones de pequeños productores de la región del altiplano de Guatemala, norte y oriente del país. Se estima que son aproximadamente 30.000 agricultores en 200 comunidades de los departamentos de Chimaltenango, Sacatepéquez, Sololá, Quiché, Alta y Baja Verapaz y Jalapa principalmente.

El cultivo de la papa en Guatemala es realizado por casi 26.000 productores, los cuales en total siembran alrededor de 7.000 hectáreas (CABI, 2011). El país tiene una alta productividad en el cultivo de este tubérculo, superior al promedio mundial, de 36 contra 17 toneladas métricas por hectárea. El $90 \%$ de la producción nacional es para consumo interno y el resto se exporta a países de Centroamérica. Las importaciones son mínimas, menos de $1 \%$ del consumo total.

La producción de tomate se encamina mayoritariamente (85\%) al consumo interno. Las exportaciones se destinan, casi en su totalidad, al país vecino de El Salvador. El área cosechada aumentó 25\% en el período 2006 a 2011, en tanto que la producción se mantuvo estable, lo que implicó una reducción en la productividad de 43,1 a 34,6 toneladas métricas por hectárea, respectivamente (Coyoy y Díaz, 2013).

El valor de las exportaciones de la agricultura a pequeña escala en 2013 fue de \$1.183,6 millones, equivalente al 11\% de estas del país (ver Tabla 2). Del total de las exportaciones agrícolas producidas a pequeñas escala, $60 \% \mathrm{co}-$ rrespondió a café y $18 \%$ a cardamomo. El restante $22 \%$ pertenece a diversas frutas, legumbres y hortalizas. Para fines de esta investigación destacan las exportaciones de tomate, con $\$ 22,5$ millones, papa con $\$ 9,3$ millones y cebolla con $\$ 4,5$ millones (CEPAL, 2013). 
Tabla 2

Cultivos agrícolas a pequeña escala

\begin{tabular}{|c|c|c|c|c|}
\hline Cultivo & $\begin{array}{c}\text { Número de } \\
\text { fincas }\end{array}$ & $\begin{array}{c}\text { Superficie } \\
\text { cultivada } \\
\text { (hectáreas) }\end{array}$ & $\begin{array}{l}\text { Extensión } \\
\text { promedio }\end{array}$ & $\begin{array}{c}\text { Exportaciones } \\
\text { Millones de } \\
\text { US\$ }\end{array}$ \\
\hline Café (cereza) & 171334 & 267905 & 1,6 & 714,5 \\
\hline $\begin{array}{l}\text { Cardamomo } \\
\text { (cereza) }\end{array}$ & 57434 & 46276 & 0,8 & 215,5 \\
\hline Tomate & 5398 & 3410 & 0,6 & 22,5 \\
\hline $\begin{array}{l}\text { Flores y } \\
\text { plantas } \\
\text { ornamentales }\end{array}$ & 5023 & 2459 & 0,5 & 66,8 \\
\hline Papa & 26984 & 6759 & 0,3 & 9,3 \\
\hline $\begin{array}{l}\text { Legumbres y } \\
\text { hortalizas } \\
\text { (total) }\end{array}$ & 10663 & 4214 & 0,4 & 155 \\
\hline Total nacional & 2001286 & 1642085 & 0,8 & 1183,6 \\
\hline
\end{tabular}

Fuente: INE, 2003; CEPAL, 2013

\section{Agricultura extensiva y pobreza}

El enfoque metodológico utilizado en este artículo consiste en contrastar el caso de dos municipios donde prevalece la agricultura extensiva, que hace las veces de grupo control, con otros dos en los cuales se practica la agricultura a pequeña escala. La idea es evidenciar las diferencias entre los primeros y los segundos en lo que respecta a pobreza y desarrollo humano.

La selección de los dos municipios con agricultura extensiva se hizo en función de que presentaran características similares a los dos de agricultura a pequeña escala. Estos últimos fueron los primeros en seleccionarse, debido al dinámico desarrollo evidenciado en el período 2000 y 2006, a través de la reducción de la magnitud de pobreza entre su población. Como ambos municipios se sitúan en regiones geográficas opuestas y difieren en su composición étnica, pero tienen en común el predominio de la agricultura a pequeña escala, se optó por seleccionar dos ubicados en regiones opuestas y con diferente composición étnica, pero con prevalencia de agricultura extensiva; la idea fue realizar el contraste de la hipótesis planteada en la investigación. Así, los dos municipios con agricultura a pequeña escala, grupo de estudio, se ubican en las regiones oriente y occidente del país, uno con mayoría de población mestiza y el otro con mayoría indígena. Los dos municipios con agricultura extensiva, grupo control, se ubican en las regiones sur y norte, el primero con mayoría de población no indígena y el segundo con una composición mestiza. 
Los de agricultura extensiva analizados son Santa Lucía Cotzumalguapa y El Estor, presentan similitudes y diferencias. En ambos predomina la agricultura extensiva, en el primer caso de caña de azúcar, y en el segundo, de banano y palma africana. Los dos tienen altos niveles de pobreza, mayores al promedio departamental y nacional (ver Tabla 3). En Santa Lucía Cotzumalguapa, la pobreza en 2006 era de 54\%, contra 59\% en 2000; en Estor era de 81\%, contra 78\%, en los periodos mencionados(Romero y Zapil, 2009).

La diferencia entre ambos municipios se da en cuanto a su ubicación geográfica y en la composición étnica. Santa Lucía Cotzumalguapa se encuentra en el sur del país, en el departamento de Escuintla, y El Estor, en la región norte, en el departamento de Izabal. En cuanto al segundo punto de divergencia, en el primer municipio la población no indígena es mayoría, mientras que en el segundo es minoría.

\begin{tabular}{lcc}
\hline \multicolumn{2}{c}{ Tabla 3 } \\
Indicadores de pobreza y desarrollo humano & $\mathbf{2 0 0 6}$ \\
\hline & Pobreza & IDH \\
\hline Santa Lucía Cotzumalguapa & $54 \%$ & 0,62 \\
\hline Depto. De Escuintla & $43 \%$ & 0,6 \\
\hline El Estor & $81 \%$ & 0,55 \\
\hline Depto. De Izabal & $51 \%$ & 0,56 \\
\hline Nacional & $50,9 \%$ & 0,64 \\
\hline
\end{tabular}

Fuente: SEGEPLAN, 2011.

El municipio de Santa Lucía Cotzumalguapa tiene una extensión territorial de 432 kilómetros. Se encuentra a 90 kilómetros de la capital de la República y a 32 de la cabecera departamental. El clima es cálido, el $64 \%$ del suelo tiene pocas restricciones y 10 ríos atraviesan el territorio del municipio, que en su mayoría es utilizado para el cultivo de caña de azúcar (Concejo Municipal de Desarrollo, 2010).

La población proyectada a 2009 fue de casi 118.000 habitantes, de los cuales el $90 \%$ pertenece al grupo étnico no indígena y $10 \%$ al indígena. La población urbana representa $57 \%$ del total y la rural, el restante $43 \%$. Al igual que en el resto del país, la población es joven: $61 \%$ es menor a los 25 años de edad. La densidad de habitantes por kilómetro cuadrado es de 273.

El nivel de pobreza en el municipio de Santa Lucía Cotzumalguapa es de $54 \%$, superior al promedio departamental, de 42\%, y similar al promedio nacional. El IDH es de 0,62, cifra un punto y medio mayor que el promedio del departamento de Escuintla. La tasa de alfabetización es de 72\%, menor a la del departamento de Escuintla y del promedio nacional, de $83 \%$ y $79 \%$ res- 
pectivamente. Con respecto a la cobertura de la educación secundaria, esta es de $28 \%$. El $85 \%$ de las casas se catalogan como formales y el $76 \%$ tiene acceso a agua potable. La tasa de mortalidad infantil es de 12 por cada mil nacidos vivos, cifra menor al promedio nacional, que es de 30 (Concejo Municipal de Desarrollo, 2010).

El uso del suelo en el municipio de Santa Lucía Cotzumalguapa en su mayoría es para cultivo de caña de azúcar. En este, opera ingenio La Unión, segundo más grande del país, con una cuota de mercado de casi $11 \%$ del total de producción de azúcar del país. En dicho sector, existen 125 fincas (Concejo Municipal de Desarrollo, 2010). El número y extensión de estas indica presencia de agricultura extensiva, con un tamaño promedio por finca de 83.500 hectáreas.

El municipio de El Estor es el más grande de los cuatro analizados, su extensión territorial es de 2.896 kilómetros cuadrados y se encuentra casi en el nivel del mar. Se ubica a 305 kilómetros de la capital de la República. El clima es húmedo tropical, con una temperatura de alrededor de 35 grados centígrados y lluvias durante la mitad del año. La mayoría del territorio (86\%) comprende cobertura boscosa, mientras un $12 \%$ corresponde a área cultivada y $2 \%$ se divide entre área habitable y cuerpos de agua (Concejo Municipal de Desarrollo, 2010).

La población proyectada para el 2009 era de casi 66.000 habitantes, de los cuales 91\% pertenece al grupo étnico indígena Q'eqchí. La densidad poblacional es de 22 individuos por kilómetro cuadrado, una de las más bajas del país. El 67\% de la población habita en el área rural, de forma dispersa debido a la extensión del municipio. En cuanto al rango edad, dos terceras partes es menor a 25 años (Concejo Municipal de Desarrollo, 2010).

La tasa de pobreza en el municipio de El Estor, como ya se dijo, aumentó de $78 \%$ a $81 \%$ en el período 2000 a 2006; es decir, es mucho mayor al promedio departamental y nacional, de $52 \%$ y $54 \%$ respectivamente. Además, la localidad presenta los menores indicadores de educación de los cuatro municipios analizados. La tasa de alfabetización es de $67 \%$ y la cobertura de la educación secundaria de solo $16 \%$. Respecto a las viviendas, solo el $58 \%$ es formal y $75 \%$ de las que están ubicadas en el área urbana tiene acceso a agua potable (Concejo Municipal de Desarrollo, 2010).

La agricultura en el municipio es de gran extensión, en especial de banano y palma africana. También, hay fincas de ganado. Una reducida parte del suelo se utiliza para agricultura a pequeña escala de maíz y frijol. El cultivo de la palma se inició en 1998 y el de la caña de azúcar, en 2002; la primera comprende 31\% del total de tierra cultivable (Díaz, 2012). 


\section{Agricultura a pequeña escala y desarrollo local}

Para evidenciar el vínculo entre agricultura a pequeña escala y desarrollo local, se analizan los municipios El Progreso Jutiapa y Almolonga. El primero se ubica en la región oriente del país, con mayoría de población no indígena. Almolonga se encuentra en el occidente, con mayoría de población indígena. El principal factor común de ambos es la producción agrícola como principal actividad económica. La segunda característica relevante es su reducción en la tasa de población con condiciones de pobreza en una cantidad significativa, de veinte puntos porcentuales en promedio, entre 2000 y 2006 (Romero y Zapil, 2009). Al 2006, ambos municipios presentaban tasas de pobreza menores e índices de desarrollo humano mayores al promedio nacional (ver Tabla 4). En efecto, la tasa de pobreza en El Progreso Jutiapa a 2006 fue de $41,6 \%$ y de $39,9 \%$ en Almolonga, cifras menores a la de $54,3 \%$ del promedio nacional y la del promedio departamental. Por su parte, el índice de desarrollo humano en cada uno de los municipios, durante ese año, fue casi 0,67 , un poco mayor al promedio nacional, de 0,64, y mayor que el departamental, que correspondió a 0,61 para Quetzaltenango, al cual pertenece Almolonga, y de 0,59 para Jutiapa, al que pertenece El Progreso Jutiapa.

Tabla 4

Indicadores de pobreza y desarrollo humano 2006

\begin{tabular}{lcc}
\hline & Pobreza & IDH \\
\hline Almolonga & 39,95 & 0,68 \\
\hline Depto. De Quetzaltenango & $44 \%$ & 0,61 \\
\hline El Progreso Jutiapa & $41,6 \%$ & 0,67 \\
\hline Dedpto. de Jutiapa & $47,3 \%$ & 0,59 \\
\hline Nacional & $54,3 \%$ & 0,64
\end{tabular}

Fuente: SEGEPLAN, 2011.

El municipio de El Progreso Jutiapa tiene una extensión territorial de 68 kilómetros cuadrados. La población al censo de 2002 fue de 22.220 habitantes (Instituto Nacional de Estadística, 2003). El 42\% de los censados durante dicho lapso era menor de 15 años, lo cual significa que la región contaba con los beneficios del bono demográfico. El clima es semicálido, con temperatura entre 19 y 24 grados centígrados. El municipio es atravesado por cuatro ríos: Ovejero, Chiquito, Colorado y Morán. Estos recursos hídricos se completan con los obtenidos de la laguna Retana, que fue drenada entre 1960 y 1963, a través de un canal de 3 kilómetros de largo, cuyo final tiene una compuerta para acumular agua empleada en riego. El drenaje de la laguna permitió a los productores agrícolas obtener más de la cosecha natural propia de la época 
de lluvia (López, 2005). Los suelos son de origen volcánico, y con vocación agrícola y forestal.

El nivel de pobreza en El Progreso Jutiapa es mucho menor que el promedio departamental, de 39,9\% contra $66,2 \%$, respectivamente, lo cual constituye un indicador de un mejor nivel de vida de sus habitantes respecto a los de municipios próximos. En el área educativa, se cuenta con una tasa de alfabetización de 92\%, mayor al promedio nacional, de 88\%, y departamental, de $77 \%$. Asimismo, tiene una tasa de cobertura a nivel secundario alta, de $36 \%$ contra $21 \%$ del promedio nacional. El $97 \%$ de la población vive en casa formal y tiene acceso a agua potable (Concejo Municipal de Desarrollo, 2011). En visita de campo a la cabecera del municipio, se observó desarrollo urbano, con calles pavimentadas, dotación de servicios públicos y casas propicias para el hábitat humano, algunas de dos niveles. También, se hizo evidente la utilización personal de vehículos motorizados.

La agricultura constituye la principal actividad productiva de El Progreso Jutiapa, emplea casi el $45 \%$ de la población económicamente activa, en especial en los cultivos de tomate, cebolla, chile pimiento, arroz, maíz y frijol. La laguna de Retana es en un importante sector de producción agrícola, a causa de la humedad que mantiene durante todo el año, la cual permite obtener dos cosechas anuales de tomate y otros cultivos. La producción también aumenta con el uso de sistema de riego por goteo y aspersión, con agua proveniente de los ríos que cruzan el municipio.

La práctica agrícola se realiza a pequeña escala e intensiva. A 2002, el 14\% de las fincas eran menores de una hectárea y $84 \%$ mayores de una hectárea pero menores de 10 hectáreas (González, 2005). Este tipo de agricultura acelera la erosión del suelo. La producción de maíz, frijol y arroz se destina al mercado nacional, mientras que la de tomate, cebolla y chile pimiento se dirige a tanto al mercado nacional como internacional (Concejo Municipal de Desarrollo, 2011). La exportación de estos productos se debe al acceso a carreteras pavimentadas y caminos transitables todo el año, como a la proximidad del municipio con El Salvador, uno de los principales países importadores de verduras, frutas y legumbres producidas en Guatemala.

Almolonga tiene una extensión territorial de 20 kilómetros cuadrados, de los cuales dieciocho constituyen laderas y montañas, y solo dos son terrenos planos. La población proyectada a 2010 era de casi 17.000 habitantes, con una densidad poblacional de 859 residentes por kilómetro cuadrado. El clima es frío, con temperaturas entre 12 y 18 grados centígrados. El municipio cuenta con diversas fuentes hídricas, debido a la existencia de nacimientos de agua. De hecho, Almolonga en idioma náhuatl significa "lugar donde mana el agua" (Godínez, 2004). Dicho sector cuenta con canales de riego, cuya fecha de construcción se desconoce. La deforestación para ampliar el área cultivable produjo la desaparición de los riachuelos Chinamá y Cañal. La vocación del suelo no es agrícola, sino edáfica forestal, motivo por el que los pro- 
ductores agrícolas utilizan fertilizantes en cantidades altas (Concejo Municipal de Desarrollo, 2010).

El porcentaje de población en condiciones de pobreza es un poco menor al promedio departamental, con $41,6 \%$ contra $44 \%$, respectivamente. La diferencia es mayor en el caso de la extrema pobreza, $4,3 \%$ y $10,0 \%$, en su orden. La tasa de alfabetización es de $81 \%$, casi diez puntos porcentuales menor al promedio del departamento. En el área de salud, la mortalidad de niños menores de un año es inferior a la de los otros municipios del departamento, 4\% contra 19\%. El $95 \%$ de la población vive en casa formal, con acceso a agua potable (Concejo Municipal de Desarrollo, 2010). En visita de campo a la cabecera del municipio, se observó desarrollo urbano, con calles pavimentadas, dotación de servicios públicos y casas propicias para el hábitat humano, un alto porcentaje de dos niveles. También se observó el uso generalizado de vehículos motorizados.

La producción agrícola es el centro de la actividad económica en Almolonga, al punto que esta región se conoce como "La hortaliza de Centroamérica" y los habitantes son llamados "Judíos de Guatemala" (Goldin, 1989), en referencia a su tradición comercial, la cual es descrita en narraciones de la época colonial, en especial por Antonio Fuentes y Guzmán en su obra "Recordación Florida" (Goldín, 2003). Según la tradición oral de los pobladores, el cultivo de hortalizas fue introducido en la década de 1940 por un misionero estadounidense. En sus inicios, la producción la compraban comerciantes de Quetzaltenango para revenderla, pero luego los productores se integran y cubren la actividad de comercialización (Godínez, 2004).

La agricultura se da a pequeña escala y de manera intensiva. Un estudio realizado en 2001 sobre una muestra de 189 productores reveló que 93 de las unidades productivas a esa fecha tenía un tamaño menor a una hectárea. El uso permanente del suelo incide en menor productividad, situación contrarrestada con un mayor uso de fertilizantes (Godínez, 2004). La producción agrícola se enfoca en verduras y hortalizas, en especial, zanahoria, cebolla, papa, repollo, lechuga, ejote y remolacha.

La falta de vocación del suelo para la agricultura impulsó el uso temprano de fertilizantes, en especial urea, alrededor de los años 1950 (Godínez, 2004). Por su parte, la construcción de la carretera Panamericana dos décadas antes creó las condiciones de infraestructura para la comercialización del producto agrícola, al permitir su transporte a menor costo; así se superaron los obstáculos de comunicación que impiden el desarrollo de comunidades agrícolas señalados por Theodore Schultz. El uso de fertilizantes y productos agroquímicos permitió experimentar la innovación tecnológica de la agricultura, también sostenida por el citado autor (González-Vega, 2005). Sin embargo, el uso intenso de estos se convierte en un problema para Almolonga, debido a la sobredosis de fertilizantes, pesticidas, herbicidas y otros insumos similares empleados en los cultivos. Además, esto ha generado la contamina- 
ción de los manantiales y fuentes hídricas (Concejo Municipal de Desarrollo, 2010).

\section{Lecciones aprendidas}

En tres de los cuatro municipios analizados, se observa desarrollo local, medido a través de la reducción en la tasa de pobreza. Este hecho es congruente con lo postulado por Schultz (1967) y coincide con la evidencia encontrada por otros autores, tales como Diao, Hazell, Resnick y Thurlow (2006), Thomson (2004), y Deithier y Effenberger (2011), cuyos hallazgos fueron mencionados en el apartado de revisión literaria. En congruencia con Mellor (2000), estudios recientes en diversos países muestran el crecimiento de la agricultura, no de la industria, como el mayor contribuyente en la reducción de la pobreza, a través de la creación de empleo. Según este autor, la evidencia es clara al indicar que se debe aumentar el crecimiento agrícola si se quiere reducir la pobreza de manera más rápida.

En los dos municipios analizados donde se practica la agricultura de gran extensión la evolución de la pobreza entre 2000 y 2006 registró un comportamiento mixto. En el municipio de Santa Lucía Cotzumalguapa se redujo cinco puntos porcentuales en ese período, en tanto que en El Estor aumentó tres. Además, estos presentan tasas de pobreza mayores al promedio departamental, con una diferencia estadística significativa.

Los datos, en este caso, señalan que la agricultura de gran extensión no contribuye a mejorar las condiciones de vida de la población, como es la situación en El Estor, o, en el mejor de los casos, tiene una reducida contribución, tal como se da en Santa Lucía Cotzumalguapa. Una posible explicación es que el empleo generado por los cultivos de caña de azúcar, banano y palma africana es temporal, lo cual no permite obtener un ingreso permanente durante el año, necesario para mejorar las condiciones de vida de la población de los municipios.

Asimismo, el incremento en la productividad en la agricultura extensiva y mecanizada genera inferior demanda de bienes y servicios, debido al menor empleo creado, lo que contribuye menos a la reducción de la pobreza (Dixon y Gulliver, 2001). Además, los salarios pagados en las plantaciones tienden a ser bajos, el pago promedio mensual en la agricultura es de casi $\$ 225,00$ (CEPAL, 2013), para una familia de cinco personas. Por otro lado, la riqueza económica generada en los municipios es apropiada más por el capital que por el trabajo, según los datos de la distribución del PIB. Así, el excedente económico de explotación bruto participa en $40,7 \%$ en el PIB, contra 30,6\% de la remuneración de los asalariados (CEPAL, 2013).

Con respecto a los dos municipios donde se practica la agricultura a pequeña escala, se redujo la pobreza entre 2000 y 2006 . Este dato no debe sorprender, el estudio de Diao, Hazell, Resnick y Thurlow (2006) presenta diversas investigaciones que concluyen que la "Revolución Verde" en Asia contribuyó 
a sacar de la pobreza a productores agrícolas a pequeñas escala; por ejemplo, en China estos se beneficiaron del rápido crecimiento de la horticultura (Banco Mundial, 2008). Asimismo, un estudio de la FAO (1995) presenta datos de reducción de la pobreza en unidades familiares agrícolas, es decir, unidades de agricultura a pequeña escala.

En Almolonga, la pobreza bajó quince puntos porcentuales y en El Progreso Jutiapa, veintitrés (ver Tabla 5). En este caso, existe una diferencia estadística significativa, al nivel de 5\%. También, hay una variación estadística significativa a dicho nivel entre los dos municipios y el promedio departamental, tanto en la tasa de pobreza como en el índice de desarrollo humano, con valores $Z$ de casi 13,0 para Almolonga y alrededor de 14,5 para El Progreso Jutiapa, en cada una de las variables indicadas.

\begin{tabular}{lccc}
\hline \multicolumn{4}{c}{ Tabla 5 } \\
& \multicolumn{2}{c}{ Indicadores de pobreza y desarrollo humano } \\
\hline & $\mathbf{2 0 0 0}$ & $\mathbf{2 0 0 6}$ & Diferencia \\
\hline Santa Lucía Cotzumalguapa & $59 \%$ & $54 \%$ & $-5 \%$ \\
\hline El Estor & $78 \%$ & $81 \%$ & $3 \%$ \\
\hline Almolongo & $66 \%$ & $51 \%$ & $-15 \%$ \\
\hline El Progreso Justiapa & $53 \%$ & $30 \%$ & $-23 \%$ \\
\hline
\end{tabular}

Fuente: Romero y Zapil, 2009.

La alta reducción de pobreza en Almolonga y EI Progreso Jutiapa puede asociarse al modelo de producción a pequeña escala orientado al mercado existente en ambos municipios. Estudios en diversos países indican que el crecimiento económico sustentado en el sector agrícola es al menos doblemente eficaz en reducir la pobreza que el crecimiento basado en otros sectores (Banco Mundial, 2008). Thomson (2004) refiere numerosas investigaciones, elaboradas en diversos países de Asia, los cuales evidencian el impacto de la "Revolución Verde" en disminuir la pobreza rural.

Además, como lo mencionan Dixon y Gulliver (2001), el aumento de productividad en las pequeñas fincas tiene mayor incidencia en la baja de la pobreza, pues las pequeñas fincas son más intensivas en mano de obra. El modelo de producción agrícola en los dos municipios analizados permite generar empleo e ingresos a su población, en especial de quienes participan más allá de la fase de producción en la cadena de valor, esto da la oportunidad de obtener una mayor proporción del excedente económico. Como ya se dijo, cifras de la distribución del PIB, según el ingreso, indican que al excedente de explotación le corresponde un porcentaje mayor que a la remuneración de los asalariados, de 40,7\% contra 30,6\%, respectivamente (CEPAL, 2013). 
Las condiciones que han posibilitado desarrollo en Almolonga y El Progreso Jutiapa consisten en adecuadas dotaciones de capital natural, físico, económico y humano. La disponibilidad de fuentes hídricas es clave en el desarrollo de la agricultura en los dos municipios. Esto se complementa con carreteras y caminos pertinentes para el acceso a los mercados nacional e internacional. A lo anterior se agrega el ingreso de recursos financieros, en forma de remesas, proveniente de la población que emigró hacia Estados Unidos. En Almolonga, el monto promedio mensual de estas es de \$300,00 (Concejo Municipal de Desarrollo, 2010) y en El Progreso Jutiapa, es de alrededor de \$ 150.00 (Díaz, 2013).

En materia de capital humano resalta la alta tasa de alfabetización, tendiente a aplicar innovaciones tecnológicas en la agricultura y así obtener mayor productividad. Como lo señala Schultz (González-Vega, 2005), el agricultor que sabe aplicar conocimientos científicos produce en abundancia aunque la tierra sea pobre. El conocimiento ha permitido sustituir, lo que Schultz llama, la agricultura tradicional o de subsistencia por agricultura comercial, orientada al mercado. El estudio de Thomson (2004) presenta evidencia de países de Asia y África donde la productividad agrícola aumentó debido a la adopción de nueva tecnología entre productores a pequeña escala. También, el estudio de la FAO (1995) informa que el uso de esta tecnonología, en especial la bioquímica, contribuyó a reducir la pobreza en unidades familiares agrícolas.

La experiencia de los cuatro municipios analizados indica que la agricultura puede constituir un motor de desarrollo local, cuando se da a pequeña escala orientada hacia el mercado, tal como ocurre en Almolonga y en El Progreso. Cuando esta es extensiva, el aporte al desarrollo local puede ser reducido, como lo indica el caso de Santa Lucía Cotzumalguapa, o nulo, como en el municipio de El Estor. La evidencia estadística, la cual es significativa, indica que en Guatemala se puede contribuir a reducir la pobreza a través del desarrollo local generado mediante la agricultura, en especial, la realizada a pequeña escala. Este tipo de desarrollo es potenciado cuando se fortalece la dotación de capital de la localidad o comunidad, en sus diferentes formas, tales como físico, humano o financiero.

La idea del desarrollo local se encuentra poco extendida en el discurso sobre desarrollo en el país, y predomina el enfoque de crecimiento y derrame, en particular con visión macroeconómica, y sobre la base de los sectores industrial y agrícola a gran escala. Por ello, se considera conveniente promover un cambio en la visión del desarrollo presente en el país.

\section{Bibliografía}

Agrequima. (2012). Impacto social y económico del sector agrícola. Agrequima. Recuperado de http://agrequima.com/images/stories/pdf/160512vvff.pdf 
Albuquerque, F. (2003). Curso sobre desarrollo local. Madrid: Instituto de economía y geografía.

Banco Mundial (2008). Informe sobre el Desarrollo Mundial 2008. Agricultura para el desarrollo. Colombia: Banco Mundial, Mundi-Prensa y Mayol Ediciones. Recuperado de http://siteresources.worldbank.org/INTIDM2008INSPA/Resources/INFORME-SOBRE-EL-DESARROLLO-MUNDIAL-2008.pdf

Bejerano, J. (1998). Economía de la agricultura. Bogotá: Tercer Mundo.

CABI. (2011). Reporte del sector agro y sus perspectivas. Guatemala.

CEPAL (2013). Estudio económico de América Latina y el Caribe. Naciones Unidas. Recuperado de http://repositorio.cepal.org/bitstream/handle/11362/1085/S2013574_es. pdf? sequience $=1$

Concejo Municipal de Desarrollo. (2010). Plan de desarrollo. Almolonga. Guatemala: Segeplan.

Concejo Municipal de Desarrollo. (2010). Plan de desarrollo. El Estor. Guatemala: Segeplan.

Concejo Municipal de Desarrollo. (2011). Plan de desarrollo. El Progreso Jutiapa. Guatemala: Segeplan.

Concejo Municipal de Desarrollo. (2010). Plan de desarrollo. Santa Lucía Cotzumalguapa. Guatemala: Segeplan.

Coyoy, E. y Díaz, G. (2013). Estimación del aporte de la PYME a las exportaciones de Guatemala. Integración y Comercio, 37(17), 103-111.

Dethier, J. y Effenberger, A. (2011). Agriculture an development. A brief review of literature. Policy research working paper, (5553). Washington: World Bank.

Diao, X., Hazell, P., Resnick, D. y Thurlow, J. (2006). The role of agriculture in development: the implications for Sub-Sahara Africa. DGSD Discussion paper (29). Washington: IFPRI.

Díaz, G. (2012). Opciones de política para las inversiones agrícolas y la gobernanza de los mercados: en apoyo a la agricultura a pequeña escala en Guatemala. Guatemala: OXFAM.

Díaz, G. (2013). Desarrollo local y dotación de capital. Un análisis empírico. Revista Nova Scientia, 6(1), 258-267.

Díaz, J. y Ascolí, F. (2006). Reflexiones sobre el desarrollo local y regional. Guatemala: URL-KFW.

Dixon, J. y Gulliver, A. (2001). Sistemas de producción agropecuaria y pobreza. Como mejorar los medios de subsistencia de los pequeños agricultores en un mundo cambiante. Washington: FAO. 
FAO. (1995). Agricultura mundial hacia el año 2010. Madrid: Ediciones Mundi-Prensa.

FAO. (2014). Exportaciones. País por producto. Recuperado de: http://faostat.fao.org/site/342/default.aspx

Goldín, L. (1989). Comercialización y cambios en San Pedro Almolonga: un caso Maya Quiché. Mayab, 5, 45-49.

Goldín, L. (2003). Procesos globales en el campo de Guatemala: opciones económicas y transformaciones ideológicas. Guatemala: Flacso.

Godínez, B. (2004). Diagnóstico socioeconómico. Municipio de Almolonga. Universidad de San Carlos de Guatemala, Guatemala.

González, R. (2005). Diagnóstico socioeconómico. Municipio de El Progreso Jutiapa. (Tesis). Universidad de San Carlos de Guatemala, Guatemala.

González-Vega, C. (2005). Significado de la obra de Theodore W. Schultz. En Grettel López y Reinaldo Herrera (Eds.) (2005). Agricultura y desarrollo económico. (3-16). San José, C.R.: Academia de Centro América.

Iarna. (2013). Los desafíos del desarrollo en Guatemala. Guatemala: Universidad Rafael Landívar.

INE. (2003). XI censo de población. Guatemala: Instituto Nacional de Estadística.

INE. (2004). Censo agropecuario. Guatemala: Instituto Nacional de Estadística.

INE. (2010). Encuesta nacional de empleo e ingresos. Guatemala: Instituto Nacional de Estadística.

López, P. (2005). Sistematización de experiencias de uso de tecnologías en el cultivo de tomate en la laguna de Retana, El Progreso Jutiapa. (Tesis). Universidad de San Carlos de Guatemala.

Meier, G. y Stiligtz. J. (2001). Fronteras de la economía del desarrollo. El futuro en perspectiva. Bogotá: Alfaomega.

MAGA. (2011). El agro en cifras 2011. Ministerio de Agricultura, Ganadería y Alimentación. Recuperado de http://web.maga.gob.gt/wp-content/uploads/pdf/uip/oct13/29/elagroencifras2011.pdf

Mellor, J. (2000). Elements of a poverty reduction strategy for Guatemala. Washington: USAID.

Ministerio de Economía (2010). Programa de desarrollo económico desde lo rural. SEGEPLAN. Recuperado de http://www.segeplan.gob.gt/downloads/pder/seguimiento/Informe_Junio_2010.pdf

Organización Internacional del Café (2014). Países exportadores. Disponible en: http://www.ico.org/prices/po.htm 
Pelaez, V. (2011). ¿Dónde empieza el círculo? Una mirada de género a las dinámicas territoriales rurales de la cuenca Ostúa-Güija. Revista de estudios sociales, 4(77), 71-92.

Prado, G. (1998). El pensamiento económico de Douglas C. North. LaissezFaire, 9, 13-32.

Pike, A., Rodríguez, A., y Tomaney, J. (2011). Desarrollo local y regional. Valencia: Universidad de Valencia.

Quan, G. (2007). Desarrollo Humano: una introducción conceptual. Guatemala: PNUD.

Ray, D. (2002). Economía del desarrollo. Madrid: Antoni Bosch.

Romero, W. y Zapil, S. (2009). Dinámica territorial del consumo, la pobreza y la desigualdad en Guatemala 1998-2006. Documento de trabajo No. 51, Programa Dinámicas Territoriales Rurales. Santiago de Chile: IDIESURL / RIMISP.

Rostow, W. (1961). Las etapas del crecimiento económico. México: Fondo de Cultura Económica.

Swinburn, G., Goga, S. y Murphy, F. (2006). Desarrollo económico local: un instructivo para el desarrollo y la implementación de las estrategias y planes de acción de desarrollo económico local. Banco Mundial. Recuperado de http://wwwwds.worldbank.org/external/default/WDSContentServer/WDSP/IB/2006/1 0/12/000310607_20061012153522/Rendered/PDF/337690SPANISHOPrimer01PUBLIC1.pdf

Solow, R. (1956). A Contribution to the theory of economic growth. The Quarterly Journal of Economics, 70, 65-94.

Schutlz, T. (1967). Modernización de la agricultura. Madrid: Aguilar.

Thomson, A. (2004). Agriculture, growth and poverty reduction. United Kingdom: Agriculture and Natural Resources Team.

Thirwall, A. (2003). La naturaleza del crecimiento económico. Un marco alternativo para comprender el desempeño de las naciones. México: Fondo de Cultura Económica.

Vásquez, A. (2007). Desarrollo endógeno. Teorías y políticas de desarrollo local. Investigaciones regionales, 11, 183-210.

Zimmerman, I. (1970). Países pobres, países ricos. México: Siglo XXI Editores. 